\title{
The soybean cultivar SCEL-1 shows potent anti-photoaging effects in a UV-induced three-dimensional human skin and hairless mouse model
}

Jin Woo Lee ${ }^{1+}$, Lei Peng ${ }^{1 \dagger}$, Hyun Jegal' ${ }^{1}$, No-June Park' , Sim-Kyu Bong ${ }^{1}$, Joon Won Lee ${ }^{1}$, Jeong Joo Pyo ${ }^{1}$, Yongsoo Choi ${ }^{1,2^{*}}$ and Su-Nam Kim ${ }^{1 *}$

\begin{abstract}
Soybean (Glycine max) is one of the major sources of nutrition and is used as a raw material for food and as a source of feed for livestock. The efficacy of soybeans on skin health includes their ability to reduce wrinkles and pigmentation and increase skin elasticity and moisture content. Black soybean has been consumed worldwide for a long time, especially in Korea, and is used as a medicinal food against several disorders related to the skin. To evaluate whether its effect on the skin is different based on the cultivar of soybeans, three black soybean cultivars collected in Korea, Soybean Core collection Elite Line-1 (SCEL-1), Chung Ja-3 (CJ-3) and Won Heuk (WH), were selected to compare their effect on improving photoaging induced by ultraviolet rays (UVs). We found that SCEL-1 exhibited the best efficacy among the three cultivars tested, and treatment with this soybean extract significantly reduced the expression of matrix metalloproteinase-1 (MMP-1), preventing the degradation of collagen in a 3D human skin model. In addition, SCEL-1 application improved wrinkle- and photoaging-related symptoms, such as epidermal thickening, collagen deficiency and immune cell infiltration, in an animal model established by UV irradiation. Procyanidin B2 and epicatechin isolated from the SCEL-1 cultivar inhibited MMP-1 biosynthesis in UVB-irradiated human dermal fibroblasts, and these two major components are likely related to more significantly attenuated skin photoaging. Therefore, our results indicated that SCEL-1 exhibits good anti-wrinkle effects compared to the other two black soybean cultivars, suggesting that it represents an excellent agent for anti-photoaging.
\end{abstract}

Keywords: Glycine max, SCEL-1, Photoaging, MMP-1, 3D human skin model

\section{Introduction}

Soybean (Glycine max) is one of the major nutritional sources belonging to the Fabaceae family and is cultivated globally, primarily in temperate regions [1]. Soybean contains high-quality protein, healthy oil, and low carbohydrates and has been used as a raw material for food

\footnotetext{
*Correspondence: yongsoo.choi@kist.re.kr; snkim@kist.re.kr

${ }^{\dagger}$ Jin Woo Lee and Lei Peng contributed equally to this work

${ }^{1}$ Natural Products Research Institute, Korea Institute of Science and Technology (KIST), 679 Saimdang-ro, Gangneung, Gangwon-do 25451, Republic of Korea

Full list of author information is available at the end of the article
}

and as a source of feed for livestock using suitable cultivars [2]. Many studies conducted over the past 30 years have reported that soybeans have various health-related benefits, especially for the prevention and treatment of chronic diseases. For example, soybeans lower the risk of cardiovascular disease, improve bone health, lower the risk of breast and prostate cancer, ameliorate kidney disease, relieve menopausal symptoms, and improve cognitive function and mental health [3-10].

Soybean and its functional components have a good effect on skin health, and the effects of soybeans on the skin are known to reduce wrinkles and pigmentation and 
increase skin elasticity and moisture content [11-15]. Soybeans contain active constituents, such as phenolic acids, including syringic, ferulic, and sinpaic acids; isoflavones that act as both phytoestrogens and selective estrogen receptor modifiers (SERMs); small proteins that act as protease inhibitors; and proanthocyanidins [1, 16]. These ingredients have been reported to have antiinflammatory, depigmentation, elastin and collagen fiber production, UV protection, and prevention of lipid peroxidation effects on the skin [1].

Glycine max, a cultivated soybean, has a variety of shapes, colors, sizes, and components, depending on genetic diversity. Since soybean is an important crop with a very high utilization value in the food and livestock feed industries, data on the genetic resources of more than 20,000 kinds of soybeans exist, including elite lines and wild soybeans from various countries, such China, Japan, and Korea, and are preserved in the USDA Soybean Germplasm Collection in the United States [17]. In Korea, various soybean resources have also been collected and analyzed to establish a unique soybean core group. Black soybean, which features yellow or green cotyledons in black seed coats, has been consumed worldwide for a long time, especially in Korea, and is used as a medicinal food against several disorders [18].

In this study, to evaluate whether the effect on the skin is different depending on the cultivar of soybeans, 3 black soybean cultivars collected in Korea, Soybean Core collection Elite Line-1 (SCEL-1), Chung Ja-3 (CJ3 ) and Won Heuk (WH), were selected to compare their effects on attenuating photoaging induced by UVB. As a result, it was confirmed that the SCEL-1 cultivar exhibited remarkable anti-photoaging efficacy compared to the other two species and contained procyanidin B2 and epicatechin, demonstrating potent efficacy for reducing expression of matrix metalloproteinase-1 (MMP-1).

\section{Materials and methods Plant materials}

Three black soybean cultivars, SCEL-1, CJ-3 and WH, were donated by the National Institute of Crop Science in Korea. The three soybeans were grown in a field in Paju, Korea, from May to October 2017. Soybeans were air-dried and stored at $10{ }^{\circ} \mathrm{C}$ in the dark until use. All cultivated plants were managed by essentially normal agricultural practices according to the standard protocol of the Rural Development Administration (http://www. nongsaro.go.kr/), including irrigation, fertilizer application, and pest control.

\section{Extraction}

Soybean extraction was performed according to a previous report with slight modification [19]. Dried soybeans were ground into find powder, passed through a 60 mesh sieve, and $3 \mathrm{~g}$ of the resulting powder was mixed with $70 \%$ aqueous ethanol and sonicated for $15 \mathrm{~min}$. Then, the solution containing soybean powder was retained at room temperature in the dark for approximately $14 \mathrm{~h}$. Finally, the solution was sonicated again for $15 \mathrm{~min}$. The extracted solution was passed through filter paper (Hyundai Micro, Size 300 mm, Grade No. 100, Seoul, South Korea), and ethanol in the extracted solution was evaporated using a SpeedVac evaporator (Thermo Fischer Scientific, Model SPD2010-220, Waltham, MA, USA). The remaining solution was completely dried using a freeze dryer machine (Operon, model FDCF-12003, Gimpo, Gyeonggi-do, South Korea). The final extracted powder was stored at $4{ }^{\circ} \mathrm{C}$ until use.

\section{Three dimensional human skin and cells}

A three-dimensional (3D) full thickness model of human skin $\left(E^{2} i D e r m F T^{\mathrm{TM}}\right.$ ) was purchased from MatTek Corporation (Ashland, MA, USA). The skin equivalent was maintained in Dulbecco's modified Eagle (DME)-based medium supplied by the manufacturer (EFT-300-MM) at $37{ }^{\circ} \mathrm{C}$ and $5 \% \mathrm{CO}_{2}$. Normal human dermal fibroblasts (HDFs) were obtained from Lonza (Walkersville, MD, USA) and cultured in Dulbecco's modified Eagle's medium (DMEM; Invitrogen, Carlsbad, CA, USA) supplemented with $10 \%$ fetal bovine serum (FBS; HyClone Laboratories Inc., Logan, UT, USA) and 1\% penicillin/ streptomycin (Invitrogen) at $37^{\circ} \mathrm{C}$ and $5 \% \mathrm{CO}_{2}$.

\section{UV irradiation}

A fluorescent UVB lamp (Sankyo Denki G15T8E, Sankyo Denki, Japan) with an emission spectrum between 280 and $360 \mathrm{~nm}$ at a peak of $312 \mathrm{~nm}$ was used as a UV light source. Human skin equivalents were treated with each reagent for $1 \mathrm{~h}$, irradiated with UVB at $40 \mathrm{~mJ} / \mathrm{cm}^{2}$ and subsequently changed to reagents in serum-free medium for $72 \mathrm{~h}$. Cells were seeded into 6-well plates, incubated for $24 \mathrm{~h}$, washed with PBS, and serum-free medium was added. After $24 \mathrm{~h}$, cells were pretreated with each reagent for $1 \mathrm{~h}$ before UV irradiation. The total energy dose of UVB irradiation was set to $20 \mathrm{~mJ} / \mathrm{cm}^{2}$ to optimize cell viability and MMP-1 stimulation. Thereafter, cells were irradiated with UVB and treated with reagents in serumfree medium for $48 \mathrm{~h}$.

\section{Enzyme-linked immunosorbent assay (ELISA)}

Supernatants from skin equivalents were harvested and subjected to ELISA. MMP-1 and procollagen typeI secretion were quantified from supernatants using a human MMP-1 and procollagen type-I ELISA Kit (R\&D Systems, Minneapolis, MN, USA). Relative levels of MMP-1 and procollagen type-I were normalized to 
corresponding cell viability as measured by MTT assay (Ez-cytox, Dail Lab Service. Co., Seoul, Korea).

\section{Animal experiments}

SKH-1 hairless mice (female, seven weeks old) were purchased from Orient Bio Inc. (Seongnam, Korea). All experimental procedures involving animals complied with the Guide for the Care and Use of Laboratory Animals of the National Institutes of Health (NIH Publication No. 85-23, 2011 revision). Procedures were approved by the Institutional Animal Care and Use Committee (IACUC) of Korea Institute of Science and Technology (Certification No. KIST-2016-011). SCEL-1, CJ-3, and WH $(25,50$ or $100 \mathrm{mg} / \mathrm{kg} /$ day $)$ in vehicle solution $(0.5 \%$ carboxymethylcellulose) were orally administered to hairless mice for 8 weeks under UV exposure conditions. UV irradiation protocols were performed as follows. During the first week, a UV dose of 1 minimal erythema dose (MED) corresponding to $100 \mathrm{~mJ} / \mathrm{cm}^{2}$ was applied to the backs of hairless mice. The intensity was increased by 1 MED every 2 weeks for up to 8 weeks. At the end of the experiment, mice were irradiated with 4 MEDs.

\section{Generation of replicas and image analysis}

An image of the back skin of each mouse was acquired immediately after the experiment was over. Then, replicas of dorsal skin from sacrificed mice were obtained using SILFLO resin (Cuderm, Dallas, TX, U.S.A.). Image analysis of the replicas was performed by the New Drug Development Center of Daegu-Gyeongbuk Medical Innovation Foundation (Daegu, Korea). The degree of wrinkling was evaluated using shadow analysis by Visioline VL650 (Courage + Khazaka, Köln, Germany), which contained a camera, stage and LED light source shining at a $35^{\circ}$ incident light angle to the stage. The degree of wrinkles is expressed as the mean from a factor defined as the mean ratio of width to length of all shadows considered wrinkles. The value 0 indicates a perfect circle, and 1 indicates a perfect line. Therefore, the closer a value is to 1 , the more effective it is at preventing wrinkles [20].

\section{Tissue preparation for microscopy and histology}

Mouse dorsal skin samples were fixed in 3.7\% formaldehyde solution and embedded in paraffin blocks. Serial sections were obtained using a microtome and dried and stained with several dyes, such as hematoxylin and eosin (H\&E), Masson trichrome (MT) and toluidine blue (TB). Histological appearance was examined using an Olympus CX31/BX51 microscope (Olympus Optical Co., Tokyo, Japan) and TE-2000U camera (Nikon Instruments Inc., Melville, NY, USA). Epidermal thickness was evaluated by measuring the length from the stratum corneum to the stratum basale using a ruler equipped on the microscope and the LAS v4.8 program (Leica Microsystem, Herbrugg, Switzerland).

\section{Analysis of isoflavones and other flavonoids}

The dried extract was dissolved in DMSO at $5 \mathrm{mg} / \mathrm{ml}$, and quantitative analysis of 5 major isoflavones in each extract was performed using an Agilent 1200 HPLC system (Agilent Technologies, Santa Clara, CA, USA) equipped with a G1315A diode array detector (DAD) at $254 \mathrm{~nm}$. Chromatographic separation was achieved using a Luna C18 reverse-phase column $(4.6 \times 150 \mathrm{~mm}, 5 \mu \mathrm{m}$, Phenomenex, Torrance, CA, USA) and two solvent systems, A (100\% water containing $0.05 \%$ formic acid) and B (100\% acetonitrile containing $0.05 \%$ formic acid), at a $1 \mathrm{ml} / \mathrm{min}$ flow rate. A gradient was used starting from 10 to $100 \%$ B for $30 \mathrm{~min}$. Quantification of 5 major isoflavones was performed based on the UV peak area of the daidzin standard combined with the molar extinction coefficients, and eight-point calibration curves were prepared for daidzin at concentrations from 0.01 to $10 \mu \mathrm{g} /$ $\mathrm{ml}$.

The dried extract was dissolved in $1 \mathrm{mg} / \mathrm{ml}$ in DMSO containing $2 \mu \mathrm{g} / \mathrm{ml}$ phloridzin as an internal standard, and quantitative analysis of two flavonoids in each extract was performed using a Q-Exactive benchtop hybrid quadrupole-orbitrap mass spectrometer (Thermo Fisher Scientific, Waltham, MA, USA) equipped with Vanquish ultrahigh-performance liquid chromatography (Thermo Fisher Scientific, Waltham, MA, USA). Five microliters of injected sample were separated using a Kinetex $\mathrm{C} 18$ reverse-phase column $(2.1 \times 100 \mathrm{~mm}, 2.6 \mu \mathrm{m}, 100 \AA$, Phenomenex, Torrance, CA, USA) and two solvent systems, A ( $95 \%$ water and $5 \%$ acetonitrile; $0.1 \%$ formic acid) and B (5\% water and $95 \%$ acetonitrile; $0.1 \%$ formic acid), at a flow rate of a $0.35 \mathrm{ml} / \mathrm{min}$. A linear gradient was used as follows: $3 \% \mathrm{~B}$ for $1 \mathrm{~min}, 3$ to $100 \% \mathrm{~B}$ for $10 \mathrm{~min}$, and $100 \%$ B for $2 \mathrm{~min}$. The column was maintained at room temperature and was re-equilibrated for at least $5 \mathrm{~min}$ between analyses. The separated sample was ionized by supplying $+3.2 \mathrm{kV}$ high voltage to a heated-electrospray ionization (HESI) source with sheath gas 42 and aux gas 10 for ionization stabilization. The ionized sample was moved through a transfer capillary tube at $320^{\circ} \mathrm{C}$, and all moved ions were detected using the Top 10-dependent acquisition (DDA) mode. The detailed parameters of DDA were as follows: In full MS, data were acquired at a resolution of 70,000, and ions were transferred by an autogain control (AGC) target of 1e6, max injection time ( $\max$ IT) of $100 \mathrm{~ms}$ and scan range of $150-2000 \mathrm{~m} / \mathrm{z}$. MS2 data were acquired under a resolution of 17,500 , AGC target of 2e5, max IT of $50 \mathrm{~ms}$, isolation window and normalized collision energies (NCEs) of 2.0 and $30 \mathrm{~m} / \mathrm{z}$. Procyanidin B2 and epicatechin were quantified 
based on the calibration curve of ten different concentrations from 0.01 to $1 \mu \mathrm{g} / \mathrm{ml}$ using the MS peak area ratio of each compound to the internal standard.

All peaks produced by HPLC were processed using ChemStation software (Rev. B.02.01-SR1, Agilent Technology, Santa Clara, CA, USA), and raw data produced by LC-MS/MS were processed by Qual Browser software provided by the Xcalibur package (Thermo Fischer Scientific, Waltham, MA, USA, ver.4.1.3). Standard chemicals were purchased from Sigma-Aldrich (St. Louis, MO, USA).

\section{Immunoblotting}

Whole supernatants from cells were precipitated with $10 \%$ trichloroacetic acid, and cell lysates were prepared in lysis buffer (Cell Signaling Technology, Danvers, MA, USA) containing a protease inhibitor (Roche, Penzberg, Germany) and a phosphatase inhibitor cocktail (SigmaAldrich). Then, the protein concentration of each sample was determined using the QuantiPro ${ }^{\mathrm{TM}} \mathrm{BCA}$ assay kit (Sigma-Aldrich). Proteins in the supernatants and lysates were electrophoresed on 8\% SDS-PAGE gels and transferred to polyvinylidene fluoride membranes (Millipore, Billerica, MA, USA). Then, membranes were probed with anti-MMP-1 (Calbiochem, MA, USA) primary antibody for $24 \mathrm{~h}$ at $4{ }^{\circ} \mathrm{C}$ and detected with corresponding horseradish peroxidase-conjugated secondary antibodies at room temperature for $1 \mathrm{~h}$. Chemiluminescence was detected using SuperSignal ${ }^{\circledR}$ West Femto Maximum Sensitivity Substrate (Pierce, Rockford, IL, USA) and visualized using an iBrtight FL1500 (Invitrogen, CA, USA).

\section{Statistical analysis}

The data are expressed as the means \pm SDs. Differences between the mean values in the two groups were analyzed using one-way analysis of variance (ANOVA) and Tukey's multiple comparisons post hoc analysis. $\mathrm{P}<0.05$ was considered statistically significant.

\section{Results}

SCEL-1 extract inhibits UV-induced expression of MMP-1 in a 3D human skin model

MMP-1, also known as collagenase 1, is a major collagen digesting enzyme that causes collagen degradation in UV-exposed human skin [21]. To evaluate the inhibitory effects of SCEL-1, CJ-3 and WH extracts on collagenase, we measured expression levels of MMP-1 in a 3D human skin model. UVB irradiation resulted in a threefold increase in levels of MMP-1 compared to the nonirradiated control group. However, levels of MMP-1 were significantly decreased in response to SCEL-1, CJ-3 and WH treatment in a dose-dependent manner compared to the UVB irradiation alone group. Furthermore, MMP-1 levels were decreased the most in response to application of SCEL-1 (Fig. 1a).

Type 1 collagen is a staple component of structural skin proteins and functions to maintain the integrity and tension of the dermis produced by fibroblasts [22]. Therefore, we examined the effects of SCEL-1, CJ-3 and WH on preventing collagen degradation in a $3 \mathrm{D}$ skin model. UVB irradiation reduced the amount of type I procollagen by $22 \%$ compared to the nonirradiated control group. However, levels of type I procollagen were significantly increased in response to SCEL-1, CJ-3 and WH treatment in a dose-dependent manner compared to the UVB-irradiated group, and once again, SCEL-1 was the most effective cultivar (Fig. 1b). These results indicate

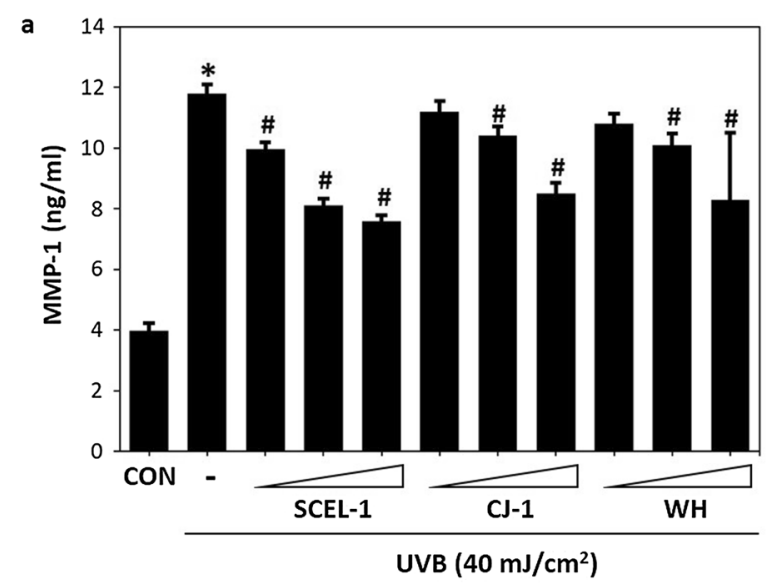

b

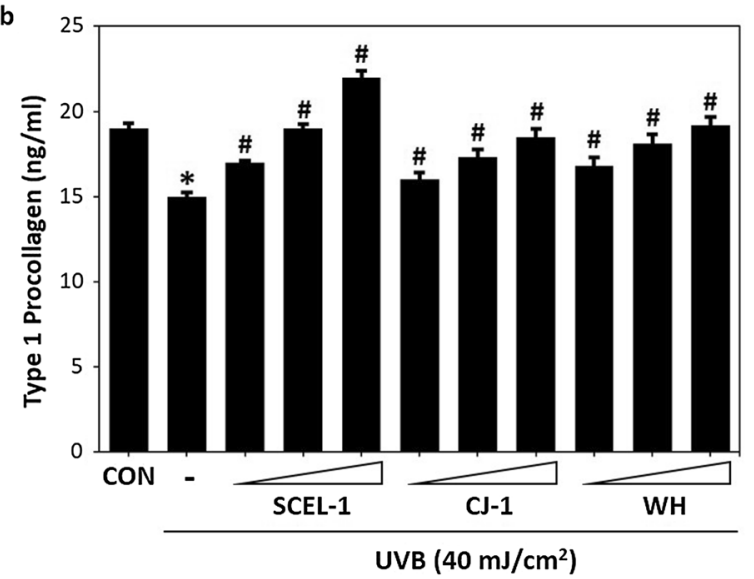

Fig. 1 SCEL-1 extract inhibits UVB-induced expression of MMP-1 and degradation of collagen in a 3D full-thickness model of human skin. Effects of SCEL-1, CJ-3 and WH on MMP-1 production (a) and procollagen type 1 (b) in a UVB-irradiated $\left(40 \mathrm{~mJ} / \mathrm{cm}^{2}\right)$ 3D human skin model. Skin tissues were treated with the indicated concentrations 10,30 or $100 \mu \mathrm{g} / \mathrm{ml}$ ) of extracts for $72 \mathrm{~h}$, and then supernatants of the skin tissue were collected for ELISA. Statistical significance of the results is given as ${ }^{*} p<0.05$ vs. control (CON) and $\# p<0.05$ vs. the UVB-irradiated group 
that SCEL-1 has the best efficacy among the three cultivars tested, and treatment with soybean extracts significantly reduces expression of MMP-1 induced by UVB, thus preventing the degradation of collagen.

\section{SCEL-1 extract prevents UV-induced wrinkle formation in hairless mice}

Next, we investigated whether SCEL-1, CJ-3, and WH can prevent wrinkle formation and skin changes in response to UV exposure in hairless mice. The 8-week schedule of UVB irradiation on the mouse was as follows (Fig. 2a). Hairless mice in the control group treated with vehicle alone did not show any significant changes in wrinkle formation over 8 weeks. However, repeated UV exposure on hairless mice increased deep or fine wrinkles on the back skin compared to the control group. In contrast, oral administration of the 3 black soybean extracts reduced wrinkle formation, and among them, the SCEL-1 extract exhibited the best anti-wrinkle effect (Fig. 2b). These results were further confirmed by image analysis using skin replicas. As a result of expressing the wrinkles as the mean form factor value, it was confirmed that the value decreased in the UV irradiated-group, but the value increased in the 3 black soybean extract-treated-groups, compared to the UV group. Consistent with previous observations, among the 3 black soybean treated groups, the value increased the most in the SCEL-1 treated group (Fig. 2c).

These results suggest that SCEL-1 treatment exhibits very strong anti-wrinkle efficacy in an animal model of wrinkle formation induced by UV irradiation.

\section{SCEL-1 extract inhibits UV-induced epidermal thickening, decreased dermal collagen and immune cell infiltration in hairless mice}

We next investigated the effect of the 3 black soybean extracts on epidermal thickness in a UVB-exposed mouse model with increasing doses of UV (1 to 4 MED) for 8 weeks. After the experiment was finished, skin samples were obtained from the dorsal skin of mice, and serially sectioned samples were stained with hematoxylin and eosin (H\&E). Epidermal thickness was increased in the UV-irradiated group by 3.5 -fold compared to the control group, but oral administration of SCEL-1 decreased the epidermal thickness to 16 and $34 \%$ in response to 25 and $100 \mathrm{mg} / \mathrm{kg} /$ day, respectively, in a dose-dependent manner compared to the UV-irradiated group. CJ-3 and WH treatment caused an approximately $20 \%$ decrease in epidermal thickness only in the $100 \mathrm{mg} / \mathrm{kg} /$ day administered group compared with the UVB-irradiated group, and their $25,50 \mathrm{mg} / \mathrm{kg} /$ day application did not exert any significant effects on epidermal changes (Fig. 3a, d).
Collagen deficiency occurs from repeated exposure to UV rays, and the decrease in collagen content in the dermis has been thought to be the cause of wrinkles on photoaged skin. Therefore, we examined whether the 3 kinds of black soybean extracts prevented decreased collagen content induced by UV exposure in skin by Masson's trichrome (MT) staining. As shown in MT-stained tissue, collagen fibers colored blue were reduced in the UV-irradiated group compared to the control group. However, collagen fibers were increased in the SCEL-1-, CJ-3- or WH-treated groups compared to the UV-irradiated group, and the SCEL-1-treated group exhibited a higher collagen boosting effect than the other two groups (Fig. 3b, e).

UV exposure to skin increases the number of infiltrating mast cells in the dermis, the release of histamine and the release of inflammatory substances from the skin. The blue dot in the dermis is toluidine blue-stained mast cells, and this blue spot increased in the UV-irradiated group compared to the control group, while the oral application of the SCEL-1, CJ-3 and WH extracts for 8 weeks decreased the infiltrated mast cell number compared to UV irradiation Furthermore, SCEL-1 treatment showed better efficacy in reducing mast cell infiltration (Fig. 3c, f).

\section{Procyanidin B2 and epicatechin isolated from the SCEL-1 cultivar inhibit MMP-1 biosynthesis in UVB-irradiated human dermal fibroblasts}

Through animal experiments, we confirmed the beneficial effect of black soybean extract on wrinkle formation and unwanted skin changes. Therefore, we next determined which components in the SCEL-1 cultivar improved the status of UV-irradiated skin. It is well known that isoflavones and other flavonoids in soybeans have antioxidant and anti-inflammatory properties in vivo and in vitro [1]. For the analysis of isoflavones, five major isoflavones, daidzin, 6"-O-malonyldaidzin, 6"-malonylgenistin, daidzein, and genistin, were quantified using LC-DAD at $254 \mathrm{~nm}$ based on the UV peak area of the daidzin standard combined with the molar extinction coefficients, and the quantitative results in three different black soybean cultivars are summarized in Table 1. The results showed that there was no significant difference in the five major isoflavones among the three different cultivars used in this study (Fig. 4a). However, a significant difference in the contents of two flavonoids, proanthocyanidin B2 and epicatechin, measured by LC-MS was clearly observed among the three different cultivars. SCEL-1 contained 17- to 30-fold higher levels of proanthocyanidin B2 and epicatechin than the other soybean cultivars, CJ-3 and WH (Fig. 4b, c). From the quantitative analysis of isoflavones and two major antioxidants, proanthocyanidin B2 
a

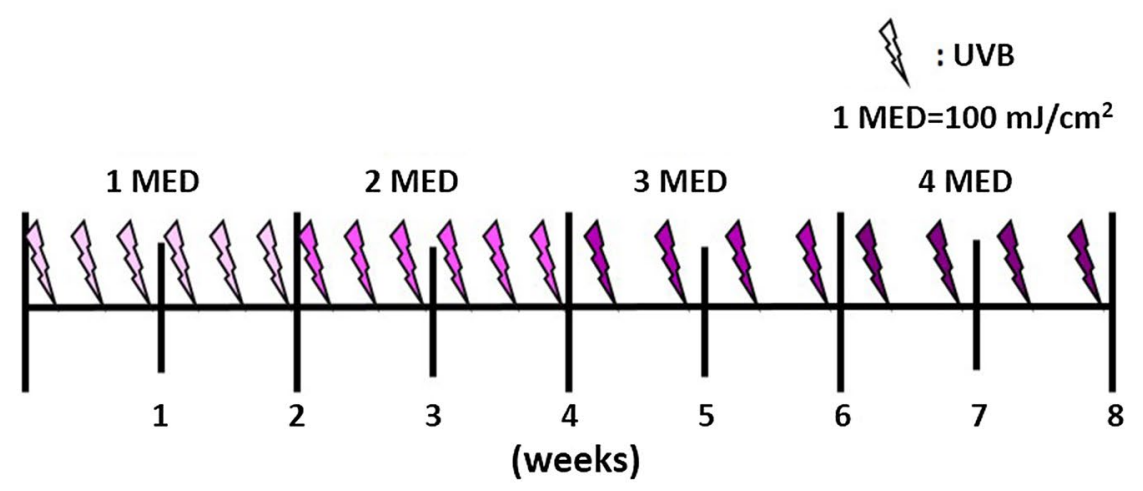

b

UVB

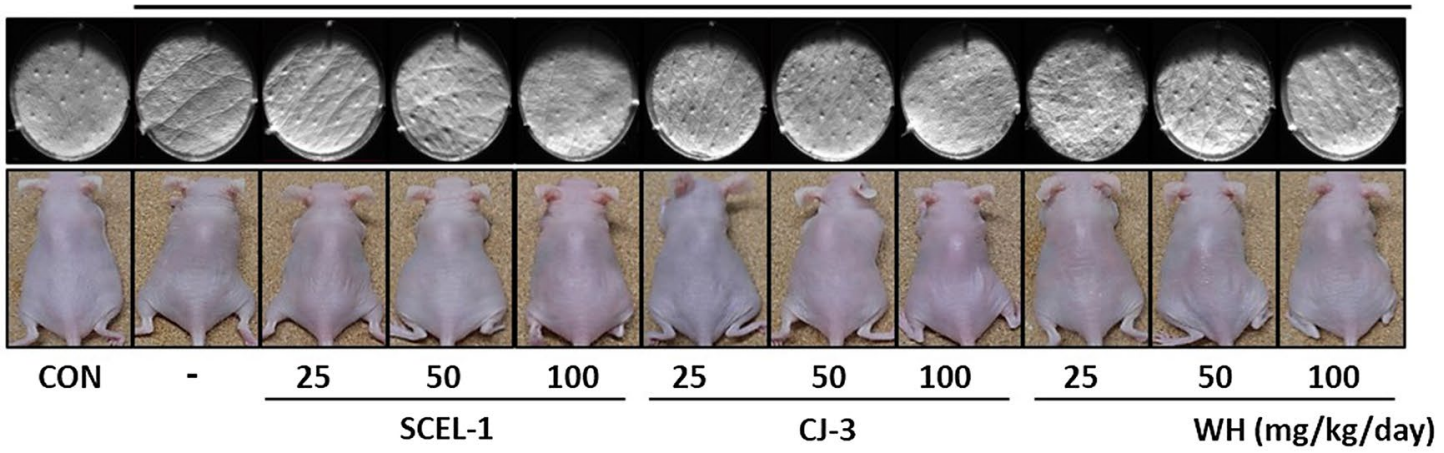

C

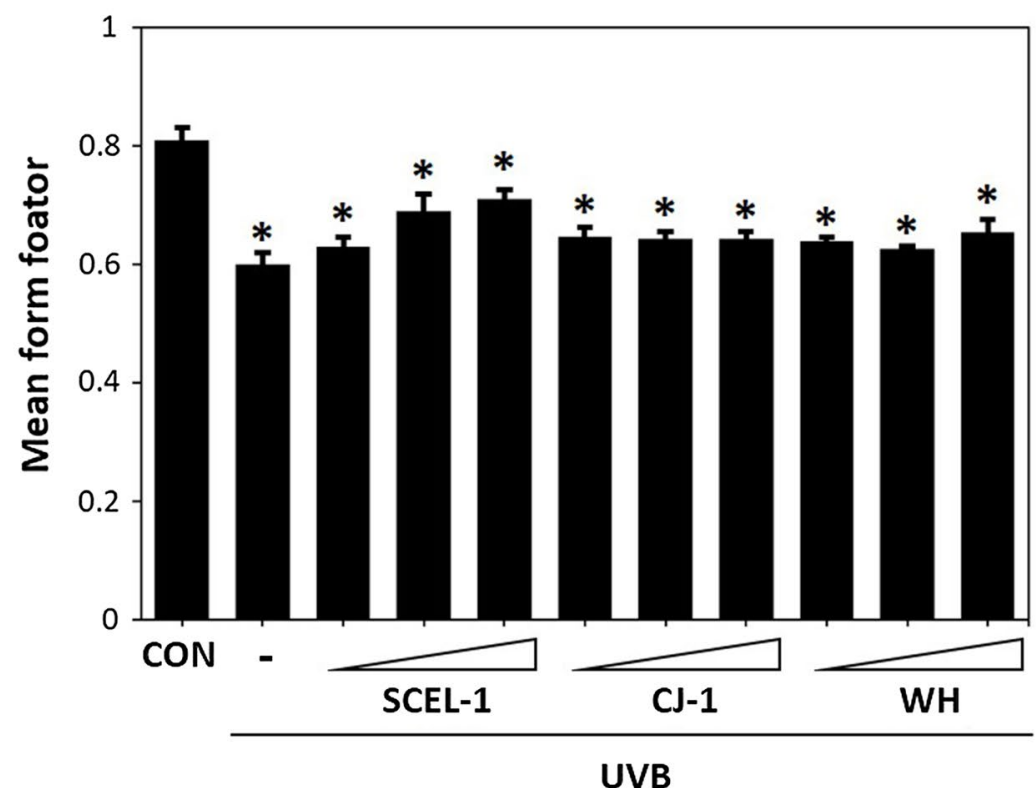

Fig. 2 SCEL-1 extract prevents UVB-induced wrinkle formation in hairless mice. a The dorsal skin of hairless mice was irradiated with UVB three times per week for the first 4 weeks and two times per week for the subsequent 4 weeks. The UV dose was increased every 2 weeks by 1 MED ( 1 MED $=100 \mathrm{~mJ} / \mathrm{cm}^{2}$ ) up to 4 MEDs. (b) Indicated soybean extracts ( 25, 50 or $100 \mathrm{mg} / \mathrm{kg} /$ day) were applied to the backs of hairless mice $2 \mathrm{~h}$ before UV irradiation. After 8 weeks of repeated UV treatment, UV-exposed dorsal areas (bottom panel) and skin replicas (top panel) were imaged. c To quantitatively assess skin wrinkling, a visiometer was used to analyze replica surfaces. Results are expressed as the mean \pm SD $(n=7)$ for the parameter mean form factor. Statistical significance for the results is given as ${ }^{*} p<0.05$ vs. control (CON) and ${ }^{*} p<0.05$ vs. the UVB-irradiated group 


\section{a}

UVB

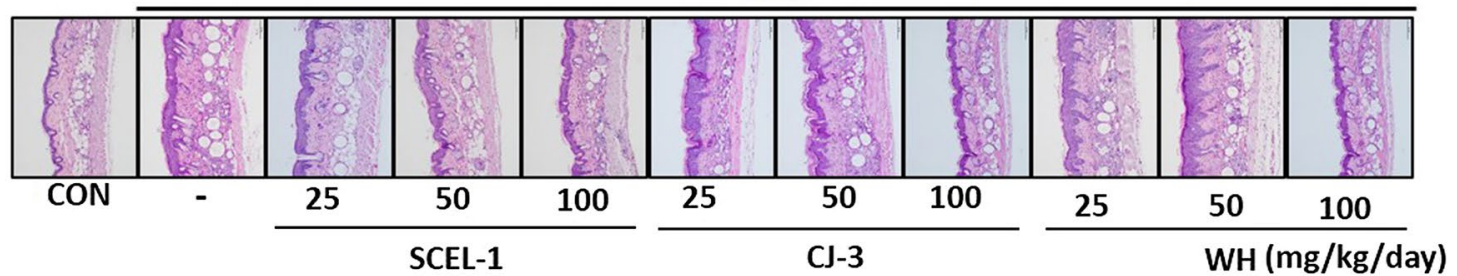

b

UVB

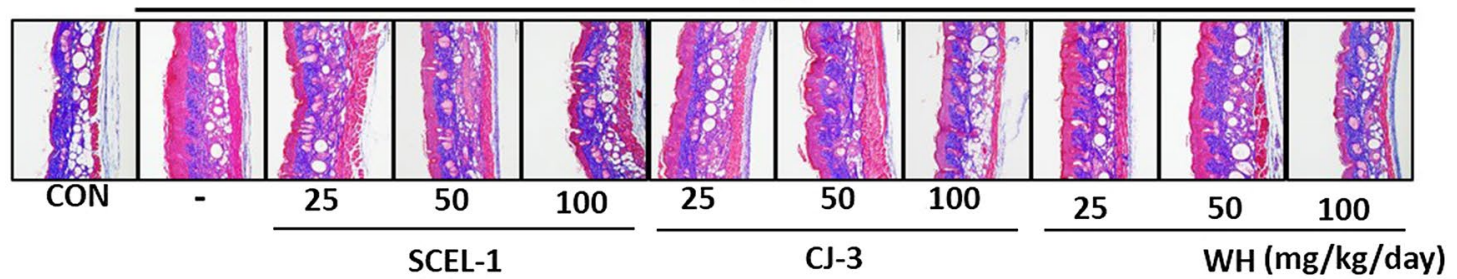

C

UVB

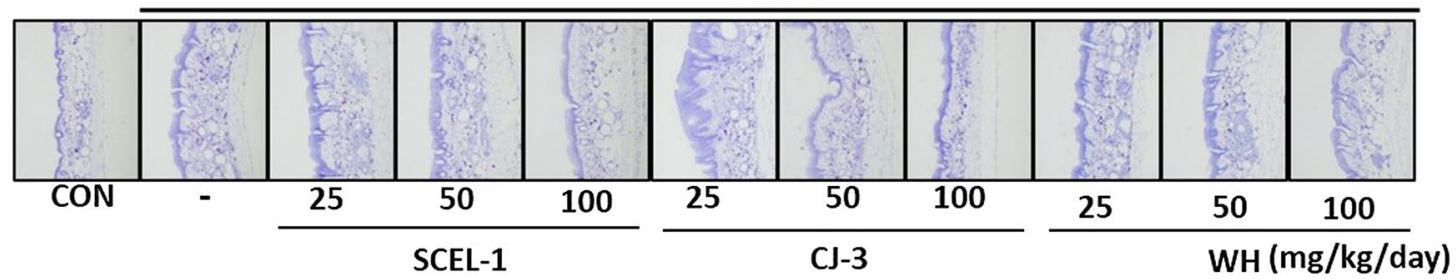

d

e
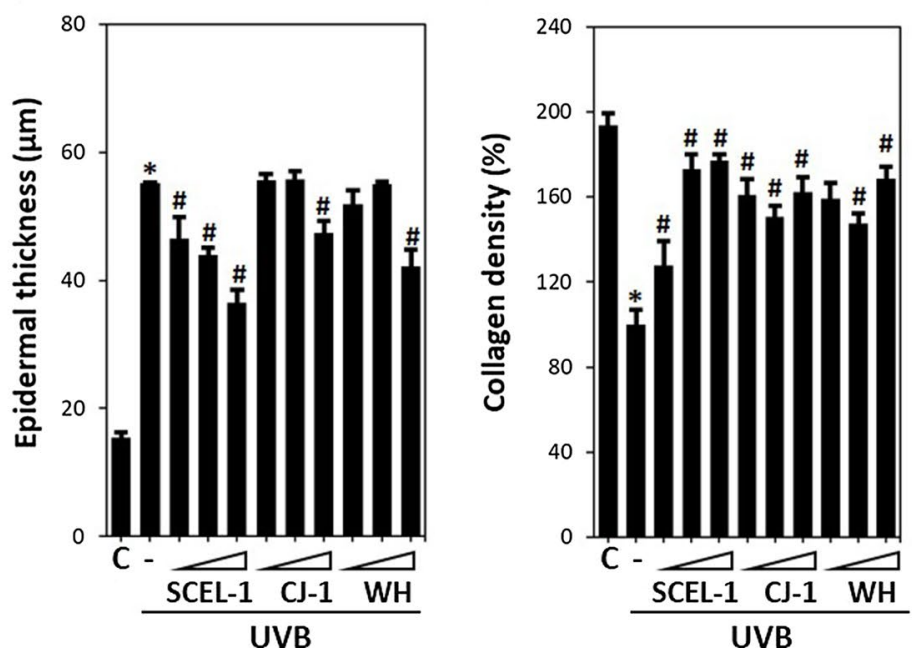

f

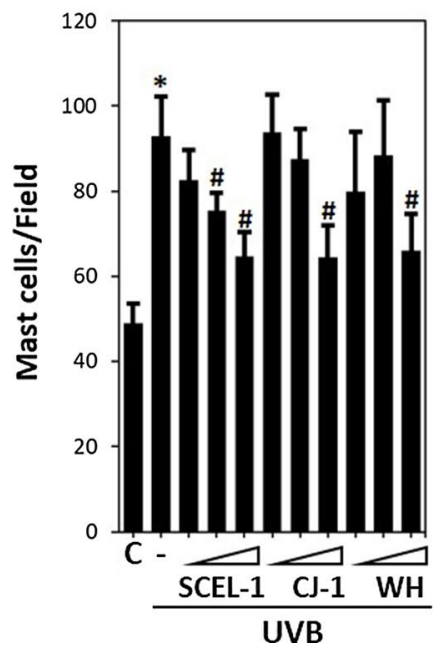

Fig. 3 SCEL-1 extract inhibits UV-induced epidermal thickening, dermal collagen deficiency and immune cell infiltration in hairless mice. Hairless mice were orally treated with the indicated concentrations ( 25, 50 or $100 \mathrm{mg} / \mathrm{kg} /$ day) of SCEL-1, CJ-3 and WH extracts as described in "Materials and methods". After 8 weeks of repeated UV irradiation, animals were sacrificed, and dorsal areas were biopsied. Serial sections (4 $\mu \mathrm{m})$ were mounted onto silane-coated slides and stained with H\&E (a), MT (b) and TB (c). Epidermal thickness (d), collagen density (e) and mast cell number $(\mathbf{f})$ are expressed as the mean \pm SD $(n=7)$ compared to vehicle controls. Statistical significance for the results is given as ${ }^{*} p<0.05$ vs. control $(\mathrm{CON}$ or $\mathrm{C})$ and $\# p<0.05$ vs. the UVB-irradiated group 
Table 1 Comparing of dietary phytochemicals in soybean extracts of 3 cultivars

\begin{tabular}{|c|c|c|c|c|c|c|c|}
\hline \multirow[t]{3}{*}{ Cultivars } & \multicolumn{7}{|l|}{ Contents $(\mathrm{mg} / \mathrm{g})$} \\
\hline & \multirow{2}{*}{$\begin{array}{l}\text { Pro-anthocyanidins } \\
\text { Procyanidin } \mathrm{B2}^{\mathrm{a}}\end{array}$} & \multirow{2}{*}{$\begin{array}{l}\text { Flavanols } \\
\text { Epicatechin }^{\mathrm{a}}\end{array}$} & \multicolumn{5}{|l|}{ Isoflavones } \\
\hline & & & Daidzin $^{b}$ & 6-Malonyldaidzin & 6-Malonylgenistin ${ }^{b}$ & Daidzein $^{b}$ & Genistin $^{b}$ \\
\hline SCEL-1 & $4.77 \pm 0.46$ & $3.98 \pm 0.51$ & $1.31 \pm 0.078$ & $6.06 \pm 0.45$ & $3.58 \pm 0.18$ & $0.4 \pm 0.18$ & $0.08 \pm 0.11$ \\
\hline CJ-3 & $0.27 \pm 0.083$ & $0.239 \pm 0.059$ & $0.835 \pm 0.15$ & $4.31 \pm 0.18$ & $4.39 \pm 0.18$ & $0.23 \pm 0.21$ & $0.235 \pm 0.21$ \\
\hline WH & $0.286 \pm 0.059$ & $0.126 \pm 0.061$ & $0.935 \pm 0.12$ & $4.46 \pm 0.28$ & $4.01 \pm 0.23$ & $0.37 \pm 0.18$ & $0.31 \pm 0.19$ \\
\hline
\end{tabular}

${ }^{a}$ Contents were analyzed by mass spectrophotometry analysis

${ }^{\mathrm{b}}$ Contents were analyzed by high performance liquid chromatography

and epicatechin, it could be hypothesized that two major antioxidants, proanthocyanidin B2 and epicatechin, are likely related to more significantly attenuated skin photoaging in response to the SCEL-1 cultivar.

To investigate the effects of two major compounds in SCEL-1 on collagenase, the major skin degradation enzyme, we measured MMP-1 protein levels in human dermal fibroblasts (HDFs). Results showed that proanthocyanidin B2 (Fig. 5a, c) or epicatechin (Fig. 5b, d) significantly inhibited MMP-1 biosynthesis in UVBirradiated HDFs. Taken together, our data indicate that procyanidin B2 and epicatechin, which are specific components found in the SCEL-1 cultivar, decrease the expression of MMP-1, showing efficacy in attenuating wrinkles.

\section{Discussion}

Photoaging is a noticeable premature skin aging condition caused by prolonged and repeated exposure to UV light, which is linked to a series of physiological and pathological processes and is characterized by fine and coarse wrinkles, along with a combination of skin lags, tissue changes, roughness, dryness, and impaired skin barrier function [23-25].

Black soybeans exert positive effects on skin care. In this study, the effect of three black soybean cultivar extracts on wrinkle improvement was confirmed using in vivo and in vitro experiments. Among the 3 soybean extracts, SCEL-1 exerted the best anti-wrinkle effect, which was demonstrated by reducing expression of MMP-1 and inhibiting degradation of collagen in the 3D human skin model (Fig. 1a-d). In addition, the fact that the SCEL-1 extract was superior in wrinkle improvement compared to the other two cultivars was also confirmed in the skin of a hairless mouse model irradiated with UV (Fig. 2b, c). Considering the staining results of the skin tissue, it was confirmed that administration of SCEL-1 exhibited the best improvement in epidermal thickness, collagen density, and the number of infiltrating mast cells among extracts of the three cultivars (Fig. 3a-d).
Taken together, the above results show that the effects of SCEL-1 have the best wrinkle improvement among the three black soybean cultivars. Analysis of the three black soybean cultivars showed that they contained similar amounts of isoflavones but different amounts of procyanidin B2 and epicatechin (Table 1, Fig. 4a, b). These two ingredients were 17 to 30 times higher in SCEL-1 than in the other two cultivars, and the difference in the content of these two components was predicted to mediate the difference in wrinkle improvement efficacy. Therefore, we investigated the wrinkle improvement effect of these compounds, which was in accordance with MMP-1 production in a UV-irradiated HDF cells. The application of procyanidin $\mathrm{B} 2$ or epicatechin decreased the expression of MMP-1 in UV-exposed HDFs (Fig. 5a-d), indicating that it suppresses the formation of wrinkles by preventing the breakdown of collagen molecules. Collagen loss in the dermal extracellular matrix due to decreased synthesis or irregular degradation that changes the amount and quality of the skin constituents is a major cause of natural aging- and photoaging-driven wrinkles [26, 27]. Oxidative stress is another important factor that induces skin photoaging $[28,29]$. ROS lead to cross-linking, inhibition of biosynthesis and damage to collagen fibers [30]. The two components identified as the primary components of SCEL-1, procyanidin B2 and epicatechin, were reported to have a cell protective effect against UVB by inhibiting oxidative stress and preventing collagen decomposition by reducing expression of MMP [31]. These reports support our results that SCEL-1 is effective at attenuating wrinkle photoaging.

In summary, we confirmed that SCEL-1 exhibited the best efficacy among the three cultivars tested, and treatment with this soybean extract significantly reduced the expression of matrix metalloproteinase-1 (MMP-1), preventing the degradation of collagen in a 3D human skin model. In addition, SCEL-1 application improved wrinkle- and photoaging-related symptoms, such as epidermal thickening, collagen deficiency and immune cell infiltration, in an animal model established by UV 
a
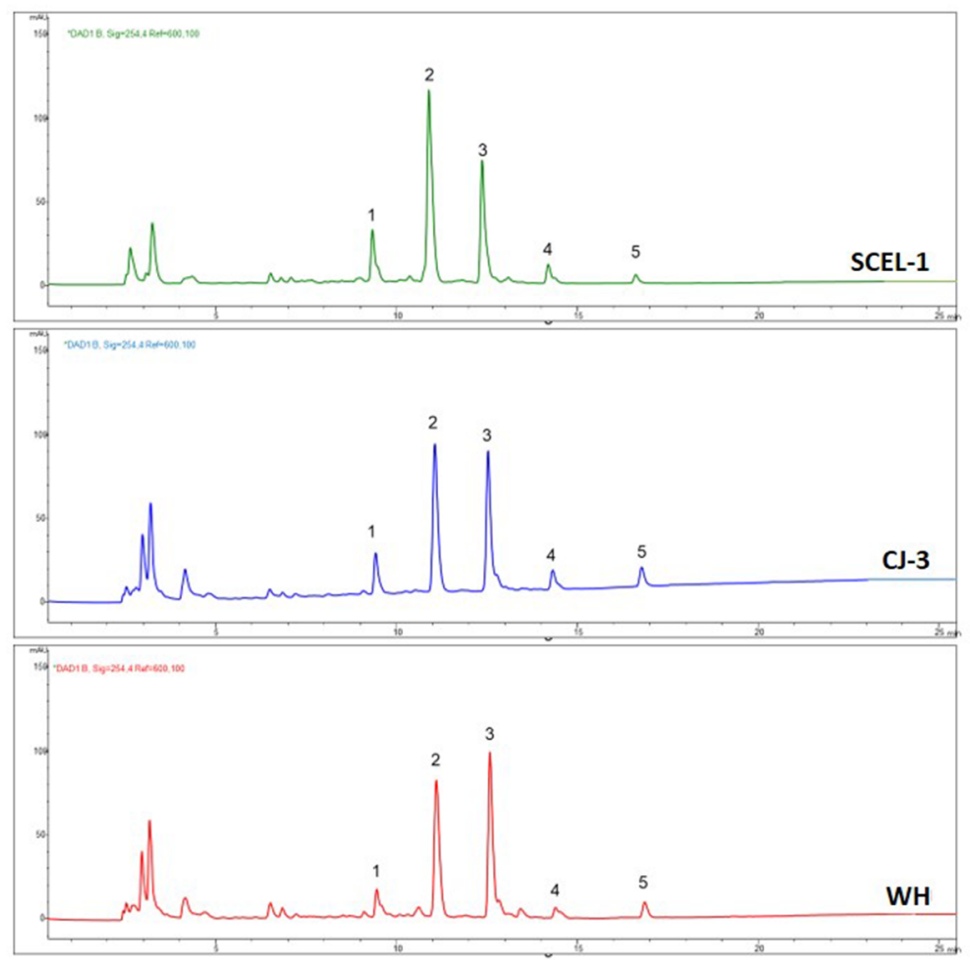

b
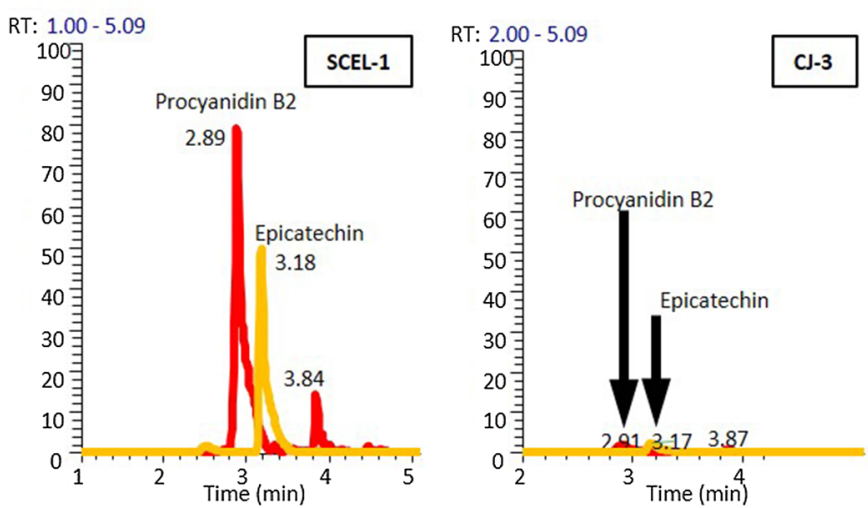

RT: $2.00-5.09$

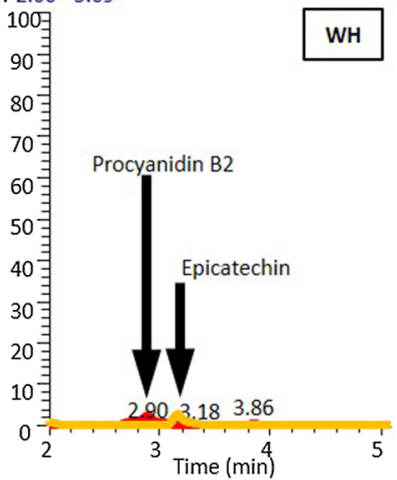

C<smiles>Oc1cc(O)c2c(c1)O[C@H](c1ccc(O)c(O)c1)[C@H](O)[C@H]2c1c(O)cc(O)c2c1O[C@H](c1ccc(O)c(O)c1)[C@H](O)C2</smiles>

Procyanidin B2<smiles>Oc1cc(O)c2c(c1)O[C@H](c1ccc(O)c(O)c1)[C@H](O)C2</smiles>

Epicatechin

Fig. 4 Chromatograms of major isoflavones, procyanidin B2 and epicatechin and chemical structures. a Three kinds of soybean extracts were analyzed using high-performance liquid chromatography. Five total peaks were identified by references. The identified isoflavones were daidzin (1), 6"-O-malonyldaidzin (2), 6"-malonylgenistin (3), daidzein (4), and genistin (5). b Three kinds of soybean extracts were analyzed using high-resolution mass spectrometry. Procyanidin B2 (m/z 449.12) and epicatechin (m/z 291.09) contained in SCEL-1, CJ-3 or WH are expressed by extracted chromatogram (XIC). The intensity of XIC was fixed at 4e6, and the XIC window was $\pm 0.01 \mathrm{Da}$. c Chemical structures of procyanidin B2 and epicatechin 
a

MMP-1

GAPDH

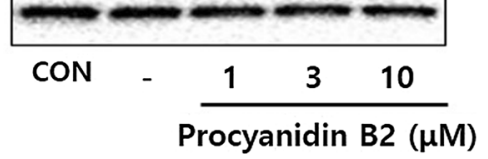

C

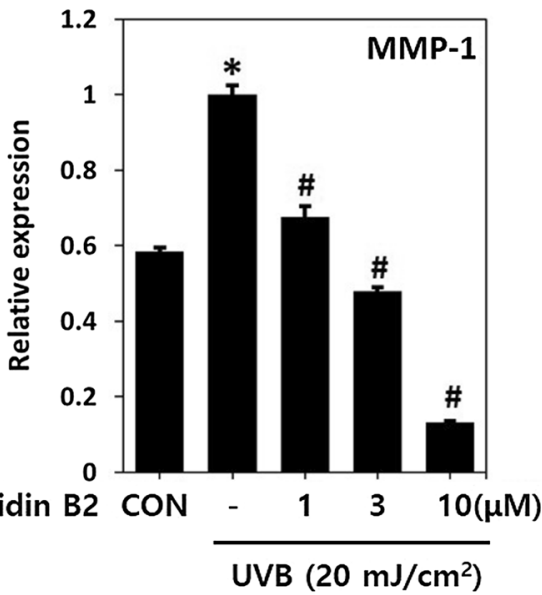

b

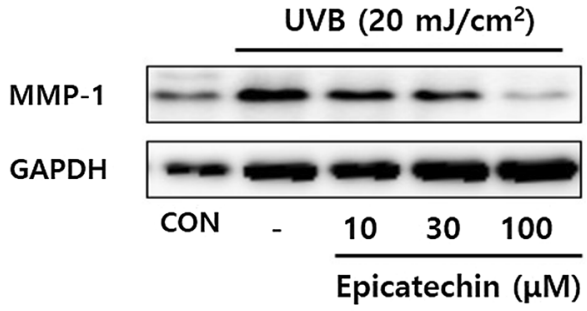

d

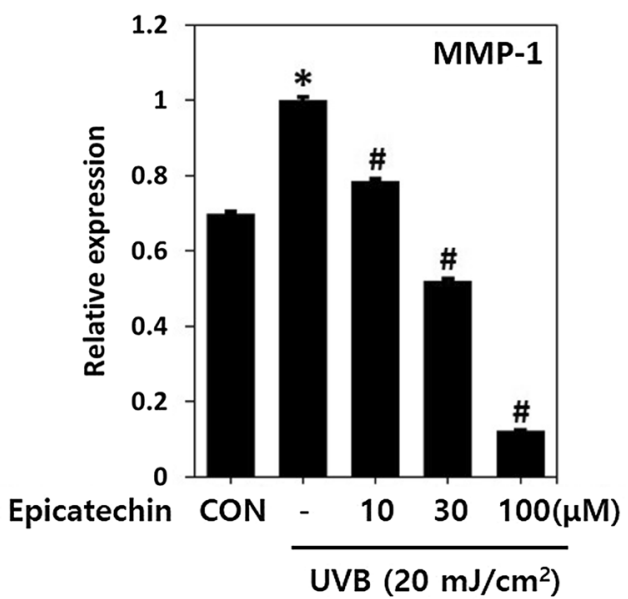

Fig. 5 Procyanidin B2 and epicatechin isolated from SCEL-1 inhibited UV-induced expression of MMP-1 in HDFs. HDFs were treated with procyanidin B2 (1, 3 or $10 \mu \mathrm{M})(\mathbf{a}, \mathbf{c})$ or epicatechin $(10,30$ or $100 \mu \mathrm{M})(\mathbf{b}, \mathbf{d})$ in combination with UVB $\left(20 \mathrm{~mJ} / \mathrm{cm}^{2}\right)$ for $48 \mathrm{~h}$ to measure MMP-1 expression. Relative MMP-1 protein expression was determined by western blot analysis. Statistical significance for the results is given as ${ }^{*} p<0.05 \mathrm{vs}$. control (CON); ${ }^{p} p<0.05$ vs. the UVB-irradiated group

irradiation. Procyanidin B2 and epicatechin isolated from the SCEL-1 cultivar inhibited MMP-1 biosynthesis in UVB-irradiated human dermal fibroblasts, and these two major components are likely related to more significantly attenuated skin photoaging. Therefore, our results showed that SCEL-1 exhibits stronger anti-wrinkle effects compared to the other two black soybean cultivars, suggesting that it represents an excellent anti-photoaging agent.

\section{Acknowledgements}

Not applicable.

\section{Authors' contributions}

JWL, LP and SNK designed the study. JWL, JWL, HJ, NJP and SKB performed the experiments. JWL and JJP analyzed the data and interpreted the results, and JWL, YC and SNK drafted the manuscript. All authors read and approved the final manuscript.

\section{Funding}

This work was supported by a grant from the Korea Institute of Science \& Technology, Republic of Korea (Grant Nos. 2Z06481, 2V08150).

Availability of data and materials Not applicable.

\section{Declarations}

Competing interests

All authors declare no conflict of interest.

\section{Author details}

${ }^{1}$ Natural Products Research Institute, Korea Institute of Science and Technology (KIST), 679 Saimdang-ro, Gangneung, Gangwon-do 25451, Republic of Korea. ${ }^{2}$ Division of Bio-Medical Science and Technology, KIST School, University of Science and Technology, Seoul 02792, Republic of Korea.

Received: 8 November 2021 Accepted: 11 January 2022 Published online: 21 January 2022

\section{References}

1. Waqas MK, Akhtar N, Mustafa R, Jamshaid M, Khan HM, Murtaza G (2015) Dermatological and cosmeceutical benefits of Glycine max (soybean) and its active components. Acta Pol Pharm 72:3-11

2. Messina MJ (1999) Legumes and soybeans: overview of their nutritional profiles and health effects. Am J Clin Nutr 70:439s-450s

3. Pabich M, Materska M (2019) Biological effect of soy isoflavones in the prevention of civilization diseases. Nutrients 11:1660

4. Kim IS, Kim CH, Yang WS (2021) Physiologically active molecules and functional properties of soybeans in human health-a current perspective. Int J Mol Sci 22:4045 
5. Tsugane S (2020) Why has Japan become the world's most long-lived country: insights from a food and nutrition perspective. Eur J Clin Nutr 75:921-928

6. McGraw NJ, Krul ES, Grunz-Borgmann E, Parrish AR (2016) Soy-based renoprotection. World J Nephrol 5:233-257

7. Chen LR, Chen KH (2021) Utilization of isoflavones in soybeans for women with menopausal syndrome: an overview. Int J Mol Sci 22:3212

8. Ko JW, Chung YS, Kwak CS, Kwon YH (2019) Doenjang, a Korean traditional fermented soybean paste, ameliorates neuroinflammation and neurodegeneration in mice fed a high-fat diet. Nutrients 11:1702

9. Messina M (2016) Soy and health update: evaluation of the clinical and epidemiologic literature. Nutrients 8:754

10. Zou Y, Chang SK (2011) Effect of black soybean extract on the suppression of the proliferation of human AGS gastric cancer cells via the induction of apoptosis. J Agric Food Chem 59:4597-4605

11. Park H, Seo JW, Lee TK, Kim JH, Kim JE, Lim TG, Park JHY, Huh CS, Yang H, Lee KW (2021) Ethanol extract of Yak-Kong fermented by lactic acid bacteria from a Korean infant markedly reduces matrix metallopreteinase-1 expression induced by solar ultraviolet irradiation in human keratinocytes and a 3D skin model. Antioxidants 10:291

12. Hsieh MC, Wu YC, Huang SH, Kuo YR, Lee SS (2018) A single-center, randomized, double-blind, Placebo-controlled clinical trial of the effectiveness of ANT1 soybean extract cream on skin recovery after Nd: YAG laser treatment. Ann Plast Surg 80:s26-s29

13. Yan CH, Chen XG, Li Y, Han R (1999) Effects of genistein, a soybeanderived isoflavone, on proliferation and differentiation of B16-BL6 mouse melanoma cells. J Asian Nat Prod Res 1:285-299

14. Ando H, Niki Y, Yoshida M, Ito M, Akiyama K, Kim JH, Yoon TJ, Lee JH, Matsui MS, Ichihashi M (2010) Keratinocytes in culture accumulate phagocytosed melanosomes in the perinuclear area. Pigment Cell Melanoma Res 23:129-133

15. Lee S, Kim JE, Suk S, Kwon OW, Park G, Lim TG, Seo SG, Kim JR, Kim DE, Lee M, Chung DK, Jeon JE, Cho DW, Hurh BS, Kim SY, Lee KW (2015) A fermented barley and soybean formula enhances skin hydration. J Clin Biochem Nutr 57:156-163

16. Kanamoto Y, Yamashita Y, Nanba F, Yoshida T, Tsuda T, Fukuda I, NakamuraTsuruta S, Ashida H (2011) A black soybean seed coat extract prevents obesity and glucose intolerance by up-regulating uncoupling proteins and down-regulating inflammatory cytokines in high-fat diet-fed mice. J Agric Food Chem 59:8985-8993

17. Valliyodan B, Brown AV, Wang J, Patil G, Liu Y, Otyama PI, Nelson RT, Vuong T, Song Q, Musket TA, Wagner R, Marri P, Reddy S, Sessions A, Wu X, Grant D, Bayer PE, Roorkiwal M, Varshney RK, Liu X, Edwards D, Xu D, Joshi T, Cannon SB, Nguyen HT (2021) Genetic variation among 481 diverse soybean accessions, inferred from genomic re-sequencing. Sci Data 8:50

18. Tsoyi K, Park HB, Kim YM, Chung Jl, Shin SC, Shim HJ, Lee WS, Seo HG, Lee $\mathrm{JH}$, Chang KC, Kim HJ (2008) Protective effect of anthocyanins from black soybean seed coats on UVB-induced apoptotic cell death in vitro and in vivo. J Agric Food Chem 56:10600-10605

19. Xu JL, Shin JS, Park SK, Kang S, Jeong SC, Moon JK, Choi Y (2017) Differences in the metabolic profiles and antioxidant activities of wild and cultivated black soybeans evaluated by correlation analysis. Food Res Int 100(Pt 2):166-174

20. Houser T, Zerweck C, Grove G, Wickett R (2017) Shadow analysis via the C+K Visioline: a technical note. Skin Res Technol 23:447-451

21. Brennan M, Bhatti H, Nerusu KC, Bhagavathula N, Kang S, Fisher GJ, Varani J, Voorhees JJ (2003) Matrix metalloproteinase-1 is the major collagenolytic enzyme responsible for collagen damage in UV-irradiated human skin. Photochem Photobiol 78:43-48

22. Tracy LE, Minasian RA, Caterson EJ (2016) Extracellular matrix and dermal fibroblast function in the healing wound. Adv Wound Care 5:119-136

23. Pyun HB, Kim M, Park J, Sakai Y, Numata N, Shin JY, Shin HJ, Kim DU, Hwang JK (2012) Effects of collagen tripeptide supplement on photoaging and epidermal skin barrier in UVB-exposed hairless mice. Prev Nutr Food Sci 17:245-253

24. Montagna W, Kirchner S, Carlisle K (1989) Histology of sun-damaged human skin. J Am Acad Dermatol 21(5 Pt 1):907-918

25. Taylor CR, Stern RS, Leyden JJ, Gilchrest BA (1990) Photoaging/photodamage and photoprotection. J Am Acad Dermatol 22:1-15
26. Hwang E, Lee TH, Park SY, Yi TH, Kim SY (2014) Enzyme-modified Panax ginseng inhibits UVB-induced skin aging through the regulation of procollagen type I and MMP-1 expression. Food Funct 5:265-274

27. Varani J, Warner RL, Gharaee-Kermani M, Phan SH, Kang S, Chung JH, Wang ZQ, Datta SC, Fisher GJ, Voorhees JJ (2000) Vitamin A antagonizes decreased cell growth and elevated collagen-degrading matrix metalloproteinases and stimulates collagen accumulation in naturally aged human skin. J Invest Dermatol 114:480-486

28. Kim J, Lee CW, Kim EK, Lee SJ, Park NH, Kim HS, Kim HK, Char K, Jang YP, Kim JW (2011) Inhibition effect of Gynura procumbens extract on UV-B-induced matrix-metalloproteinase expression in human dermal fibroblasts. J Ethnopharmacol 137:427-433

29. Ito S, Itoga K, Yamato M, Akamatsu H, Okano T (2010) The co-application effects of fullerene and ascorbic acid on UV-B irradiated mouse skin. Toxicology 267:27-38

30. Lo YY, Cruz TF (1995) Involvement of reactive oxygen species in cytokine and growth factor induction of c-fos expression in chondrocytes. J Biol Chem 270:11727-11730

31. Liu S, You L, Zhao Y, Chang X (2018) Hawthorn polyphenol extract inhibits UVB-induced skin photoaging by regulating MMP expression and type I procollagen production in mice. J Agric Food Chem 66:8537-8546

\section{Publisher's Note}

Springer Nature remains neutral with regard to jurisdictional claims in published maps and institutional affiliations.

\section{Submit your manuscript to a SpringerOpen ${ }^{\circ}$ journal and benefit from:}

- Convenient online submission

- Rigorous peer review

- Open access: articles freely available online

- High visibility within the field

Retaining the copyright to your article

Submit your next manuscript at $>$ springeropen.com 